\title{
Lessons from repurposing HIV drugs: a prospective novel strategy for drug design
}
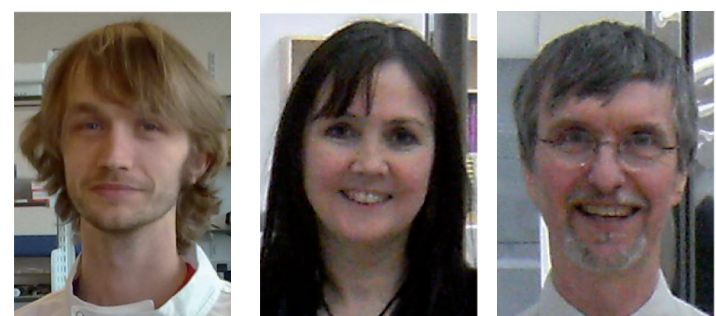

“...HIV (protease inhibitors)

may have antiviral activity

against other types

of virus."

Gavin Batman', Lynne Hampson' \& Ian N Hampson ${ }^{\dagger 1}$

'University of Manchester Viral Oncology Unit, Research Floor, St Mary's Hospital, Oxford Road.

Manchester M13 9WL, UK

†Author for correspondence: Tel.: +44 1612765606 = ian.hampson@manchester.ac.uk

It is beyond all doubt that viruses are amazing organisms. Indeed, they can be regarded as the ultimate microscopic 'smart bomb'. In comparison to a human cell, they have so little genetic material and yet they efficiently hijack numerous cellular processes in order to achieve replication. What is even more astonishing is that they often target crucial cellular functions by more than one distinct mechanism. An appropriate analogy would be 'belt and braces'. Considering the inherent restrictions on the amount of information that can be virally encoded, it is perhaps not surprising that many viruses have developed closely related approaches to take over the target organism. From the individual virus's point of view, the paradigm would be 'Why reinvent the wheel?'

The activity of the proteasome is a good example of a cellular function that is commonly targeted by many different types of virus [1]. Indeed, it is known that virtually all plant and animal viruses subvert the activity of the host proteasome in order to produce a cellular environment that is compatible with the virus life-cycle [2-4]. HIV is no exception to this rule, in that the virus also targets the ubiquitin-proteasome system [5,6].

In light of these observations, we noted that Piccinini et al. had demonstrated that specific HIV protease inhibitors (PIs) had the ability to selectively inhibit the mammalian proteasome in addition to their predicted activity against the HIV-encoded aspartyl protease [7]. Indeed, these authors also made the point that some of the antiviral properties of HIV PIs may actually rely on their ability to inhibit specific functions of the mammalian proteasome. It is important to realize that this effect was due to selective targeting of the chymotrypsin-like

activity of the proteasome, since complete proteasome blockade is highly toxic. Therefore, given the ubiquitous importance of the proteasome to the life-cycle of virtually all known viruses, we hypothesized that HIV PIs may have antiviral activity against other types of virus. In fact, there was already some evidence in support of this since various HIV PIs had been demonstrated to have activity against SARS coronavirus and avian influenza virus, in addition to several other human pathogens including bacteria, mycobacteria, fungi and even malaria [8-10]. However, the underlying rationale behind these effects was initially considered to be the ability of HIV PIs to cross-target other pathogen-encoded aspartyl proteases. In view of the findings of Piccinini et al., these effects could also be related to the ability of these compounds to selectively inhibit virally induced activation of the mammalian proteasome [11,12].

\section{“...various HIV (protease inhibitors) had been demonstrated to have activity against SARS coronavirus and avian influenza virus, in addition to several other human pathogens..."}

In view of the preceding arguments, we tested the ability of a variety of HIV PIs to block the ability of the high-risk human papillomavirus type 16 (HPV16) E6 protein to produce proteasomal degradion of the TPp53 tumor suppressor protein in cultured cervical carcinoma cells. These experiments clearly showed that indinavir and lopinavir could produce this effect, with the latter proving to be the most effective [13]. Furthermore, these compounds

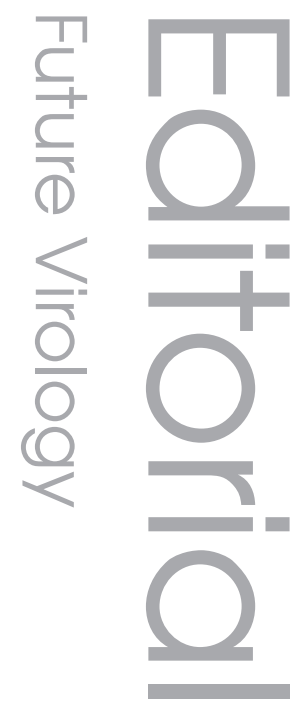

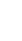

. 
were shown to induce selective apoptosis by upregulation of the antiviral protein ribonuclease $\mathrm{L}$ in these cells, which has since been confirmed in HPV16 E6/E7 immortalized keratinocytes [14]. We have also demonstrated that lopinavir has greater selective toxicity for E6/E7-expressing cells than zinc finger ejecting compounds, which are another candidate for HPV therapy [15]. Clearly, these data provide a significant body of evidence to support the use of lopinavir as a treatment for HPV-related disease, although the concentration required is more than tenfold higher than is normally achieved in cervico/vaginal fluid by oral dosing with the drug for HIV therapy [16].

In addition to antimicrobial activity, what other alternative uses have been proposed for HIV PIs? It is well known that deregulation of the proteasome is a characteristic feature of human malignant disease [17]. The importance of proteasomal activity to cancer cells is best exemplified by development of the proteasome inhibitor Velcade ${ }^{\circledR}$ (bortezomib or PS341; Millenium Pharma, MA, USA), which has proved effective for the treatment of a variety of human malignancies [18]. Since various HIV PIs cause selective inhibition of the proteasome, it is a logical assumption to predict that some of these may possess activity against malignant disease per se. One of the first indications for this came from the observed marked regression of Kaposi sarcoma (KS) in HIV patients receiving HAART. HAART can be based on combinations of nucleoside and a non-nucleoside reverse transcriptase inhibitors (NNRTI-HAART) with or without a PI (PI-HAART). Initially, regression of KS was thought to be due to immune reconstitution of AIDS patients, although the timing of the regression often preceded this. It is also highly significant that some studies demonstrated that PI-HAART was more effective at inducing a complete response of KS than NNRTIHAART [19]. It is worth noting that the development of KS is related to coinfection with $\mathrm{KS}$-associated human herpes virus 8 in AIDS patients. As previously discussed, this implies that the mode of action of HIV PIs against KS could also be related to their ability to directly target human herpes virus 8-associated functions, which, in turn, produces the observed antitumor effects $[19,20]$.

Irrespective of these antimicrobial effects, the direct antitumor effects of a variety of HIV PIs, together with their prospective mode of action, are now well established [19-21]. For example, constitutive activation of the Akt-regulated cell survival pathway is crucially important in a range of human malignancies. Akt is known to be inhibited by several HIV PIs and at a lower concentration than is required for the previously discussed effects on the proteasome [20].

In summary, the effects of these agents are both pleiotropic and concentration dependent, possessing either direct or indirect activity against a range of microbes and tumors, either as a monotherapy or acting in synergy with radiation and other cytotoxic treatments [10,19-21]. How these properties of HIV PIs are produced is clearly the subject of debate, with the following possibilities:

- Reconstitution of the immune system in HIV-positive individuals;

- Off-target activity against the mammalian proteasome;

- Off-target activity against pathogen-encoded proteases;

- Off-target activity against other host-derived, disease-associated pathways (e.g., Akt).

It is our opinion that none of the above provides a single explanation for the remarkable properties of these drugs. The more likely scenario is that it will be a combination of these that will be dependent on which particular HIV PI is being studied.

"... it is our opinion that every antiviral drug developed should be tested for activity in as wide a variety of human pathologies as is practically possible."

What is perhaps more difficult to understand is how compounds that were designed to target the HIV aspartyl protease can produce such a diverse range of effects in so many different pathologies. Our hypothesis to explain this relates to how viruses must necessarily target many of the same cellular processes. Owing to the obligate size-restricted information content of viral genomes, their proteins must be extraordinarily multifunctional, such that they can take over the host cell and achieve viral replication. Thus, it would be predicted that the selection of compounds that specifically target such pleiotropic viral proteins as the HIV aspartyl protease will yield drugs that are themselves multifunctional - an argument that is clearly supported by the preceding discussions on the various alternative uses of HIV PIs. Extending this theory further, we would suggest that 
designing novel compounds that specifically target virally encoded proteins could be used as a means of obtaining drugs with many and varied activities against a whole range of diseases. In the timeline of modern drug development activities, the identification of antivirals has only occurred fairly recently. However, for the aforementioned reasons, we feel that this area holds great promise for the development of drugs with huge potential. Indeed, it is our opinion that every antiviral drug developed should be tested for activity in as wide a variety of human pathologies as is practically possible. Furthermore, when the target disease is related to a virus and/or a malignancy, we would suggest that different combinations of these agents should also be explored, since this may help to combat compensatory overlapping ('belt and braces') functions, which are clearly present in such pathologies.

\section{Financial \& competing interests disclosure \\ The authors would like to thank The Humane Research \\ Trust, The Caring Cancer Trust and The Cancer \\ Prevention Research Trust for funding their cited work. \\ The authors have no other relevant affliations or finan- cial involvement with any organization or entity with a financial interest in or financial conflict with the sub- ject matter or materials discussed in the manuscript. This includes employment, consultancies, honoraria, stock ownership or options, expert testimony, grants or patents received or pending, or royalties. \\ No writing assistance was utilized in the production of this manuscript.}

\section{Bibliography}

1. Banks L, Pim D, Thomas M. Viruses and the $26 S$ proteasome: hacking into destruction. Trends Biochem. Sci. 28(8), 452-459 (2003).

2. Gao G, Luo H. The ubiquitin-proteasome pathway in viral infections. Can. J. Physiol. Pharmacol. 84(1), 5-14 (2006).

3. Shackelford J, Pagano JS. Targeting of host-cell ubiquitin pathways by viruses. Essays Biochem. 41, 139-156 (2005).

4. Edelmann MJ, Kessler BM. Ubiquitin and ubiquitin-like specific proteases targeted by infectious pathogens: emerging patterns and molecular principles. Biochim. Biophys. Acta 1782(12), 809-816 (2008).

5. Votteler J, Schubert U. Ubiquitin ligases as therapeutic targets in HIV-1 infection. Expert Opin. Ther. Targets 12(2), 131-143 (2008).

6. Klinger PP, Schubert U. The ubiquitin-proteasome system in HIV replication: potential targets for antiretroviral therapy. Expert Rev. Anti Infect. Ther. 3(1), 61-79 (2005).

7. Piccinini M, Rinaudo MT, Chiapello N et al. The human $26 \mathrm{~S}$ proteasome is a target of antiretroviral agents. AIDS 16(5), 693-700 (2002).

8. Yamamoto N, Yang R, Yoshinaka Y et al. HIV protease inhibitor nelfinavir inhibits replication of SARS-associated coronavirus. Biochem. Biophys. Res. Commun. 318(3), 719-725 (2004).
9. Savarino A. Expanding the frontiers of existing antiviral drugs: possible effects of HIV-1 protease inhibitors against SARS and avian influenza. J. Clin. Virol. 34(3), 170-178 (2005).

10. Bruning A, Gingelmaier A, Friese K, Mylonas I. New prospects for nelfinavir in non-HIV-related diseases. Curr. Mol. Pharmacol. 3(2), 91-97 (2010).

11. Piccinini M, Mostert M, Rinaudo MT. Proteasomes as drug targets. Curr. Drug Targets 4(8), 657-671 (2003).

12. Piccinini M, Rinaudo MT, Anselmino A et al. The HIV protease inhibitors nelfinavir and saquinavir, but not a variety of HIV reverse transcriptase inhibitors, adversely affect human proteasome function. Antivir. Ther. 10(2), 215-223 (2005).

13. Hampson L, Kitchener HC, Hampson IN. Specific HIV protease inhibitors inhibit the ability of HPV16 E6 to degrade p53 and selectively kill E6-dependent cervical carcinoma cells in vitro. Antivir. Ther. 11(6), 813-825 (2006).

14. Batman G, OLiver AW, Zehbe I, Richard C, Hampson L, Hampson I. Lopinavir up-regulates expression of the antiviral protein ribonuclease $\mathrm{L}$ in human papilloma virsi postice cervical carcinoma cells. Antivir. Ther. 16(4), 515-525 (2011).
15. Zehbe I, Richard C, Lee KF, Campbell M, Hampson L, Hampson IN. Lopinavir shows greater specificity than zinc finger ejecting compounds as a potential treatment for human papillomavirus-related lesions. Antiviral. Res. 91(2), 161-166 (2011).

16. Kwara A, Delong A, Rezk N et al. Antiretroviral drug concentrations and HIV RNA in the genital tract of HIV-infected women receiving long-term highly active antiretroviral therapy. Clin. Infect. Dis. 46(5), 719-725 (2008).

17. Schwartz AL, Ciechanover A. The ubiquitin-proteasome pathway and pathogenesis of human diseases. Ann. Rev. Med. 50, 57-74 (1999).

18. Adams J, Kauffman M. Development of the proteasome inhibitor Velcade (bortezomib). Cancer Invest. 22(2), 304-311 (2004).

19. Monini P, Sgadari C, Toschi E, Barillari G, Ensoli B. Antitumour effects of antiretroviral therapy. Nat. Rev. Cancer 4(11), 861-875 (2004).

20. Chow WA, Jiang C, Guan M. Anti-HIV drugs for cancer therapeutics: back to the future? Lancet Oncol. 10(1), 61-71 (2009).

21. Bernstein WB, Dennis PA. Repositioning HIV protease inhibitors as cancer therapeutics. Curr. Opin. HIV AIDS 3(6), 666-675 (2008). 